Review

\title{
Photochirogenesis: Photochemical Models on the Origin of Biomolecular Homochirality
}

\section{Cornelia Meinert ${ }^{1}$, Jean-Jacques Filippi ${ }^{1}$, Laurent Nahon ${ }^{2}$, Søren V. Hoffmann ${ }^{3}$, Louis d'Hendecourt $^{4}$, Pierre de Marcellus ${ }^{4}$, Jan Hendrik Bredehöft ${ }^{5}$, Wolfram H.-P. Thiemann ${ }^{5}$ and Uwe J. Meierhenrich ${ }^{1}$ *}

1 Faculté des Sciences, University of Nice-Sophia Antipolis, UMR 6001 CNRS, LCMBA, 28 Avenue Valrose, 06108 Nice, France; E-Mails: Cornelia.Meinert@unice.fr (C.M.); Jean-Jacques.Filippi@unice.fr (J.J.F.)

2 Synchrotron SOLEIL, l'Orme des Merisiers, Saint Aubin BP 48, 91192 Gif-sur-Yvette, France; E-Mail: laurent.nahon@synchrotron-soleil.fr (L.N.)

3 Institute for Storage Ring Facilities (ISA), Aarhus University, Ny Munkegade 120, DK-8000 Aarhus C, Denmark; E-Mail: vronning@phys.au.dk (S.V.H.)

4 Astrochimie et Origines, Institut d'Astrophysique Spatiale (IAS), Bât. 121, Université Paris-Sud, 91405 Orsay Cedex, France; E-Mails: Louis.DHendecourt@ias.u-psud.fr (L.H.); pierre.demarcellus@ias.u-psud.fr (P.M.)

5 Institute for Applied and Physical Chemistry, University of Bremen, Leobener Str. NW2, 28359 Bremen, Germany; E-Mails: thoralf@uni-bremen.de (J.B.); thiemann@uni-bremen.de (W.T.)

* Author to whom correspondence should be addressed; E-Mail: Uwe.Meierhenrich@unice.fr.

Received: 1 March 2010; in revised form: 26 April 2010 / Accepted: 13 May 2010 /

Published: 25 May 2010

Abstract: Current research focuses on a better understanding of the origin of biomolecular asymmetry by the identification and detection of the possibly first chiral molecules that were involved in the appearance and evolution of life on Earth. We have reasons to assume that these molecules were specific chiral amino acids. Chiral amino acids have been identified in both chondritic meteorites and simulated interstellar ices. Present research reasons that circularly polarized electromagnetic radiation was identified in interstellar environments and an asymmetric interstellar photon-molecule interaction might have triggered biomolecular symmetry breaking. We review on the possible prebiotic interaction of 'chiral photons' in the form of circularly polarized light, with early chiral organic molecules. We will highlight recent studies on enantioselective photolysis of racemic amino acids by circularly polarized light and experiments on the asymmetric 
photochemical synthesis of amino acids from only one $\mathrm{C}$ and one $\mathrm{N}$ containing molecules by simulating interstellar environments. Both approaches are based on circular dichroic transitions of amino acids that will be presented as well.

Keywords: amino acids; homochirality; enantioselective photosynthesis; asymmetric photolysis; origin of life

\section{Introduction}

Life's molecular one-handedness is an all-pervading reality on Earth: proteins are composed of lefthanded (L) amino acid monomers, while nucleic acids, the building blocks of our RNA and DNA contain exclusively right-handed (D) sugars. In contrast, organic compounds produced by non-biological processes are generally racemic, i.e., the L- and D-forms of chiral molecules are present in equal amounts. Homochirality is believed to be not only a consequence of life but probably a precondition for life, because stereo-regular structures such as protein $\beta$-pleated-sheets, which play a key role in their catalytic activity, are not able to form in the presence of equal amounts of amino acid enantiomers [1].

Prebiotic amino acids were believed to have been mainly formed in the atmosphere of early Earth, as it was successfully simulated by Urey-Miller-type experiments using electric discharges [2] and UV photons [3]. However, the reducing atmospheric conditions with methane $\left(\mathrm{CH}_{4}\right)$ used in this type of experiment are not consistent with the current geochemical and photochemical understanding of the Earth's primitive atmosphere [4,5] and it became evident that only low yields of amino acids could have been produced under non-reducing conditions in this experiment [6]. Another criticism of Miller-type experiments is that equal quantities of both amino acid forms, left- and right-handed, are produced.

A number of proposals and theories were pursued to scientifically explain how asymmetric organic compounds formed on Earth before life arose. These can be classified in two main groups: random and determinate mechanisms [1,7]. The first considers, like flipping a coin, a 50:50 chance in producing a left- or right-handed enantiomeric excess in the product formed. Hypotheses for this mechanism include the spontaneous symmetry breaking via crystallization [8] and the asymmetric adsorption on chiral mineral surfaces such as quartz [8,9]. However, these abiotic processes are supposed to be of minor efficiency for having tipped the molecular balance towards homochirality [10].

On the other hand, the tiny parity-violating energy difference between two enantiomers [11], circularly-polarized bremsstrahlung [12-14], magnetochiral photochemical effects [15-18], and the asymmetric photochemical induction of an enantiomeric excess by circularly polarized light (CPL) [19-21] are defined as potential determinate mechanisms where an external physical agent (force) induces an optical activity in the products formed. The most promising one among them appears to be the processes involving CPL, which has been proved to produce significant and reproducible enantiomeric excesses in racemic or prochiral precursors.

Several sources of CPL have been suggested, including some of extraterrestrial origin. All the more astonishing, non-racemic amino acids have been found, with the same handedness as biological amino acids have, in meteorites [22-25]-flinders of asteroids, comets, or planets-shifting the stage for the 
origin of homochirality into outer space. After some debate on possible CPL sources from pulsars [26,27] this theory recently became attractive once more with the observation of CPL in the Orion OMC-1 star formation region, reaching levels up to $17 \%$ [28-30].

Further lines of evidence that amino acids and molecules of prebiotic interest are present in space are coming from experiments mimicking primordial conditions in interstellar molecular clouds. For example, ultraviolet photoirradiation of laboratory interstellar ice analogs was shown to produce simple organic molecules, such as ethanol, formamide, nitrile, urea [31-33], and even chiral molecules such as amino acids [34-36].

In consideration of these findings, it is assumed that amino acids and other molecules spontaneously form in dense molecular clouds-the protostellar regions of space. The organic material formed subsequently may accrete together with dust and water resulting in the formation of comets [37-39]. Comets are then considered as vehicles carrying life`s molecular seeds through space $[4,40]$.

Infrared observations and meteorite sample analyses, combined with realistic laboratory interstellar ice simulations, were shown to be helpful tools in the understanding of how life's precursor molecules were probably built. These topics will therefore be discussed in this review based on the earliest fundamental breakthroughs in this field of research as well as highlighting recent discoveries.

\section{Organic Chiral Molecules in Interstellar Environments}

Since the early 1960s, scientists have suggested that extraterrestrial organic molecules hitched a ride from space to trigger life on Earth [4,41,42]. In this scenario, the evolution of life initiated more than four billion years ago when a cold, dark interstellar cloud collapsed into the swirling disk of hot gas and dust creating our Solar System.

These interstellar (dense) clouds, the birthplace of stars and planetary systems, are nowadays known to be rich in organic molecules, ions, and radicals in the gas phase. Besides large amounts of $\mathrm{H}_{2}$ and $\mathrm{He}$, more than 150 interstellar and circumstellar gaseous species, amongst which $\mathrm{CO}, \mathrm{CO}_{2}, \mathrm{NH}_{3}$, $\mathrm{H}_{2} \mathrm{CO}$, and $\mathrm{HCN}$, have been identified in the interstellar medium (ISM) by space- or Earth-based telescopes. There might be even more complex organic compounds and macromolecular matter on interstellar grains, most of them consisting of a silicate core surrounded by an icy mantle. Moreover, large molecules such as polycyclic aromatic hydrocarbons (PAHs) were observed in diffuse clouds where photochemical reactions are assumed to be the predominant processes.

Nevertheless, a detailed description of the evolution, survival, transport and transformation pathways of the ISM [39,42-45] is beyond the scope of this article, and in fact, is not completely known by now. However, we would like to highlight the main features of chemical evolution of interstellar grains to better understand their presumed contribution to the composition of comets and other extraterrestrial objects such as parent bodies of primitive carbonaceous meteorites.

Starting with "naked" silicate grains drifting in dense interstellar clouds, rapidly, condensable atomic species such as $\mathrm{C}, \mathrm{O}$, and $\mathrm{N}$ will accrete onto the surface of the nuclei and form saturated molecules such as water, methanol, and possibly simple hydrocarbons. The interstellar ice begins to form. Ultraviolet radiation from nearby stars breaks most of the chemical bonds of the frozen compounds as the icy grain grows no bigger than about ten thousands of a millimeter across. Detached molecules (radicals) recombine into larger and more complex structures. 
The main processes relevant for the formation of amino acid structures and other organic molecules include: (1) reactions in the gas phase of the cloud which result in small and volatile products due to limited reactions of single atoms or small molecules and photodestructive processes of the "unprotected" molecules [46]. (2) Reactions on the surface of the ice mantles can lead to small organic molecules which can be desorbed and/or destroyed by radiation [47]. In general, explosive desorption of an ice mantle is assumed to be the main source of organic chemicals in the gas phase of molecular clouds [48]. (3) Even organometallic chemical species can be formed due to reactions on the silicate grain surface. (4) Larger and complex organic molecules can occur within the bulk material of the mantle shielded from photodestructive processes. And finally, (5) reactions in larger clusters where desorbed reaction products will not be lost to space but remain in such an aggregate to form more complex chemical species are discussed in the literature [49].

The basic principle of these reactions is the formation of radicals out of small molecules present in the ISM induced by UV light or cosmic radiation ( $\beta$-particles, $\gamma$-rays, $x$-rays, etc.). In contact with the icy surface of dust grains, these radicals initiate further chemical reactions. However, also out of equilibrium thermal processes such as grain-grain collisions, sputtering and chemical explosion as well as grain growth processes modify interstellar dust grains in molecular clouds leading to a vast reservoir of solid state molecules in a kind of "matrix" where complexity is able to proceed.

At the time, when the dense interstellar cloud collapsed to form the Sun and the surrounding solar nebula, organic molecules from the "dressed" dust particles were incorporated into the Solar System material such as asteroids, comets and later on in planets. An interesting issue in this event is whether the Solar System preserved any chemical memory of its parent bodies which might be useful in understanding predominant processes in the pre-solar interstellar nebula (see Section 2.3).

\subsection{Amino Acid Formation in Simulated Interstellar Environments}

It is assumed that the formation of complex organic molecules such as amino acids takes place in the outer ice layers of the inorganic dust grains spread all over molecular clouds. Therefore, laboratory simulations of such growing icy organic mantles may give us some hints about the chemical composition of the building material of micrometeorites which were not incorporated in larger interstellar bodies as well as of primitive carbonaceous meteorites.

Infrared observations of the ISM allowed the precise estimation of the ice composition therein. The main components were found to be $\mathrm{H}_{2} \mathrm{O}, \mathrm{CH}_{3} \mathrm{OH}, \mathrm{CO}, \mathrm{CO}_{2}$, and $\mathrm{NH}_{3}$ [50,51]. As already outlined in the above chapter, most of the reaction pathways in these ices are triggered by UV photons and cosmic rays [52] at temperatures of about 10 to $50 \mathrm{~K}$ depending on the exact location of the ices in dense clouds.

Taking this into account, the first experiments of two independent laboratories could indeed show that the formation of amino acids in interstellar and circumstellar ices is feasible [34,35]. These experiments generally consist in the condensation of gaseous mixtures at very low temperatures $(\sim 10 \mathrm{~K})$ while an intense UV-light source induces the photochemical processing of the semi-refractory residue containing a bunch of complex organic compounds. After heating the ice to room temperature amino acid precursor structures are assumed to form with some exothermicity from re-combined radicals able 
to diffuse and react within complex radical chain reactions-not yet fully understood-in the slow melting ice [53].

The team of Bernstein et al. [35] used an initial gas mixture containing water, methanol, ammonia, and hydrogen cyanide $\left(\mathrm{H}_{2} \mathrm{O}: \mathrm{CH}_{3} \mathrm{OH}: \mathrm{NH}_{3}: \mathrm{HCN}\right)$ in molar composition of 20:2:1:1. HPLC-based analysis allowed the identification of three amino acids (glycine, serine, and alanine) in considerable quantities of a racemic nature after acid hydrolysis of the irradiated sample.

The second team [34] used a mixture of water, ammonia, methanol, carbon monoxide, and carbon dioxide $\left(\mathrm{H}_{2} \mathrm{O}: \mathrm{NH}_{3}: \mathrm{CH}_{3} \mathrm{OH}: \mathrm{CO}: \mathrm{CO}_{2}\right)$ as carbon and nitrogen precursors. They could detect 16 amino acids in the acidic hydrolyzed residue, including six proteinogenic ones (glycine, alanine, valine, proline, serine, and aspartic acid) by using enantioselective GC-MS analysis. The analytical results in this experiment were confirmed by isotopically labeled reactants to preclude any contamination by terrestrial trace compounds (Figure 1). Surprisingly, structures containing two amino groups were also detected in relatively high concentrations, such as the diamino acids diaminobutyric acid (DAB) and diaminopropionic acid (DAP), which will be of importance in the following sections.

Figure 1. Gas chromatograms showing ECEE* derivatives of ${ }^{13} \mathrm{C}$-labelled amino acids generated under simulated interstellar pre-cometary conditions. Data were obtained from GC-MS analysis after hot water extraction and $6 \mathrm{M} \mathrm{HCl} \mathrm{hydrolysis} \mathrm{of} \mathrm{VUV} \mathrm{light-processed}$ residues resulting from the condensation of $\mathrm{H}_{2} \mathrm{O}:{ }^{13} \mathrm{CH}_{3} \mathrm{OH}: \mathrm{NH}_{3}:{ }^{13} \mathrm{CO}:{ }^{13} \mathrm{CO}_{2}$ mixtures at $12 \mathrm{~K}$. Relative compositions are given on the graphs [34] (* $N$-ethoxycarbonyl ethyl esters).

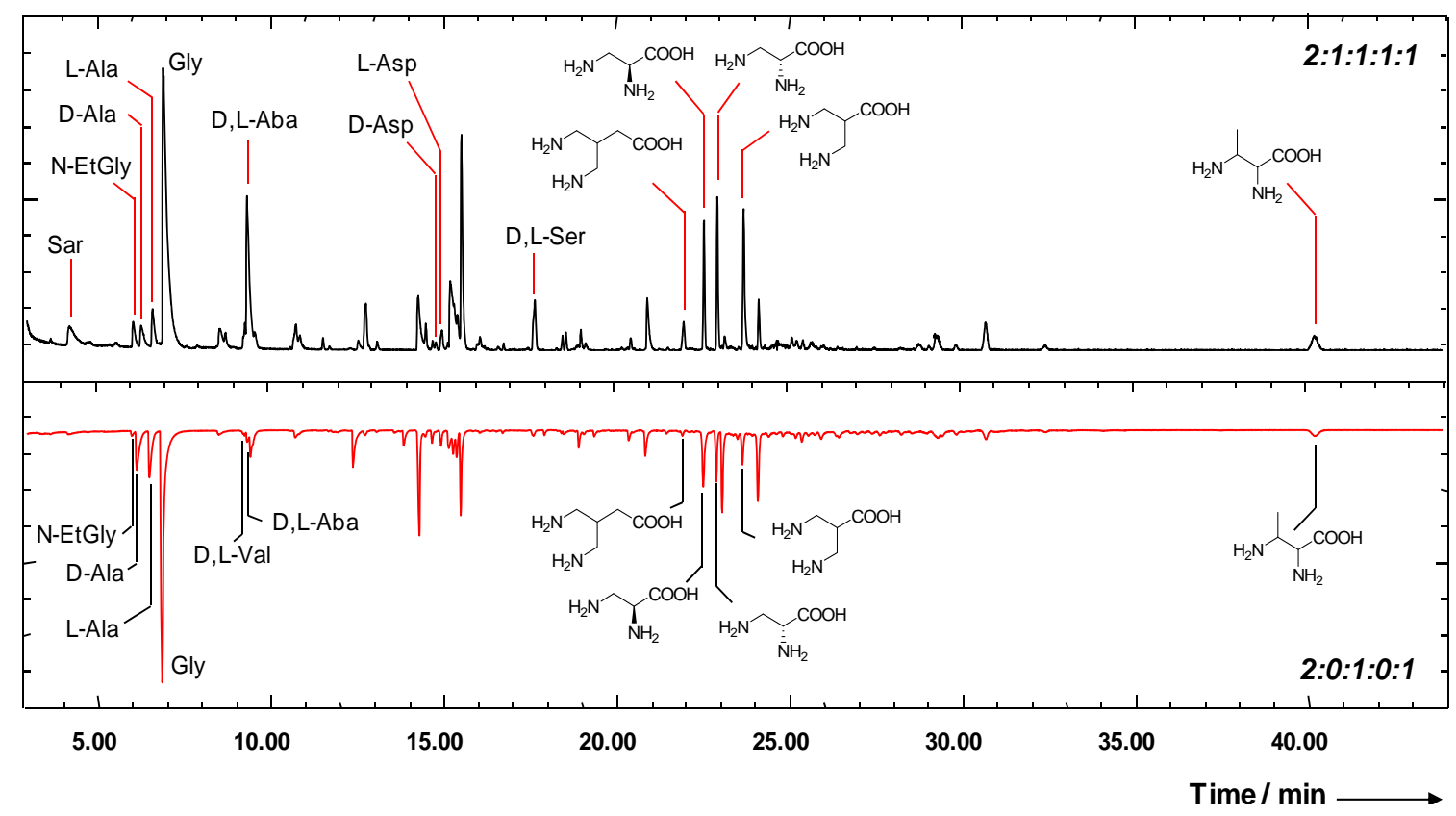

In both experiments, glycine, the simplest amino acid, was the most abundant. Moreover several amino acids identified in these interstellar ice analogs were also found in meteoritic samples [54,55]. Another remarkable observation was the increase of detected amino acids after acidic hydrolysis. This has led to the assumption that amino acids were originally present in peptidic or oligomer molecules and are released as free molecules after the additional hydrolysis step [34,56]. 
Since then, further progress in this field has been made to better understand the chemical reaction pathways [57]. Moreover, the influence on the synthesis of amino acids by different energy sources were studied using vacuum ultraviolet radiation [58,59], charged particles [60,61], cosmic rays $[56,62,63]$, high energy protons [64], or simple warm-up to room temperature experiments as well as different compositions of the starting ice mixtures [36].

In summary, a variety of photochemical reactions involving the formation of amino and diamino acids have been studied on laboratory-produced interstellar ice analogs. Ultraviolet light has been proven to be effective at promoting photochemical processes in these ices. However, the extent of these mechanisms in "real" interstellar ices is not proven, especially the lack of bright IR sources in dense clouds and the solid state of abundant molecules impede there direct observations. Further studies on these photochemical processes may provide an explanation for the occurrence of several detected organic molecules such as amino acids in different meteorites (see Section 2.2).

\subsection{Enantiomeric Enrichment in Meteoritic Amino Acids}

Although cometary nuclei and asteroids in situ analyses may soon become feasible (see Chapter 5), so far, meteorites represent the only sample of chemical evolution available for direct analyses. Their organic composition, therefore, offers a unique opportunity to answer critical questions in the field of the origin of life [65].

Carbonaceous chondrites have been formed more than four billion years ago and are believed to be primitive non differentiated bodies. They belong to a particularly primitive class of meteorites containing 2 to 3 wt. \% carbon, part of which is organic matter. They are also unique among the stony meteorites with respect to their relatively high water content. Much of our current knowledge of meteoritic organic matter has come from investigations of the Murchison meteorite, a CM2-type carbonaceous chondrite, which fell in Australia in 1969.

The first analysis of Murchison revealed that amino acids and hydrocarbons are present in this meteorite [55] and ${ }^{13} \mathrm{C} /{ }^{12} \mathrm{C}$ ratios [65] revealed that these compounds are unambiguously of extraterrestrial origin. Since then, about 500 "soluble" [66] organic compounds belonging to different classes have been identified in meteoritic samples. Among them are aliphatic amines, alcohols, aldehydes, ketones, aliphatic and aromatic hydrocarbons, mono- and dicarboxylic acids, hydroxy acids, amides, amino and diamino acids, phosphonic and sulfonic acids, and sugar-related compounds. Even molecular DNA constituents such as purine- and pyrimidine bases and their structurally-related compounds xanthine and hypoxhantine were found in the three meteorites Murchison, Murray, and Orgueil [65,67-70].

Most of the carbon content (about 70-80\%) is found, however, in the insoluble macromolecular kerogen-like fraction of the Murchison meteorite, which is however difficult to characterize. Extensive studies, in particular on amino acids, have been carried out since they are the chemical base of living organisms [54,55,71]. More than 90 diverse $\alpha-, \beta-$, and $\gamma$-acyclic and cyclic monoamino alkanoic, diamino alkanoic and alkandioic acids of up to $\mathrm{C}_{9}$ chain length have been detected in the Murchison meteorite including 13 proteinaceous amino acids (glycine, alanine, serine, threonine, methionine, phenyl alanine, tyrosine, proline, leucine, isoleucine, valine, aspartic acid, and glutamic acid) [72]. Each amino acid is present in almost all its possible constitutional isomeric forms and the abundance 
order for each carbon number is characterized as $\alpha>\gamma>\beta$, whereas branched carbon chain isomers predominateover straight ones. The most abundant amino acids are usually found to be glycine (Gly) and $\alpha$-aminoisobutyric acid (AIB) in CM chondrites, the lowest carbon number amino acid and the lowest carbon number branched $\alpha$-amino acid, respectively.

The majority of these amino acids are present as racemic mixtures, but L-enantiomer excesses were found for six $\alpha$-methylamino acids (2-amino-2,3-dimethylpentanoic acid-both diastereomers, isovaline, $\alpha$-methyl norvaline, $\alpha$-methyl valine, and $\alpha$-methyl norleucine) from the Murchison $(e e=2.8-18.5 \%)$ and Murray $(e e=1.0-6.0 \%)$ carbonaceous chondrites using enantioselective gas chromatography-mass spectroscopy [22-24]. The highest L-enantiomeric excess was found for isovaline in both $\mathrm{CM}$ meteoritic samples and as well as in Orgueil-a CI-type meteorite $(e e=15.2 \%)$. However, the high $e e_{\mathrm{L}}$ values for isovaline cannot be explained exclusively by the contribution of extraterrestrial CPL (or even terrestrial CPL) alone since the upper limit is about 10\% for a $99.99 \%$ photodecomposition rate of amino acids [73,74] demanding for an additional amplification mechanism. It is assumed that aqueous alteration on the parent body significantly influenced an amplification of higher enantiomeric excess in L-isovaline [25]. Liquid aqueous metamorphism on parents bodies of chondritic meteorites is a proven fact since the silicate material composing these meteorites show a high degree of hydration.

Another critical issue with meteoritic ee values is the risk of contamination of the analyzed meteoritic fractions with ubiquitous L-amino acids from the biosphere, which would result in overestimated enantiomeric excess values for the L-enantiomer in those samples. Since several amino acids that were found to be enantiomerically enriched are present in the terrestrial environment, with isovaline and AIB occurring in fungal antibiotics [75,76], careful attention should be paid to excluding terrestrial contamination. Compound specific stable isotope compositions of hydrogen, carbon, and nitrogen determined for several amino acids found in the meteorites demonstrated, that they are significantly isotopically enriched in the heavier stable isotopes compared to their terrestrial counterparts [24,65,77-83]. Also in this case great analytical attention is demanded since terrestrial microorganisms could metabolize extraterrestrial nutrients with their corresponding isotopes into amino acids showing a false extraterrestrial isotopic signature [76].

In conclusion, the enantiomeric excess detected in the $\alpha$-methyl amino acids strongly suggests being of interstellar origin and therefore supports the hypothesis of an extraterrestrial origin of life's homochirality. Considering that the $\alpha$-methyl amino acids in the Murchison fragment have been subject to racemization [84] and heating in the parent asteroid, the enantiomeric excess reported by the above mentioned studies may represent the lower limits of L-amino acid enrichment.

However, even if the described enantiomeric excess is shown to be relatively small compared to the almost exclusive use of L-amino acids on Earth it would be difficult to achieve these ee values by UVCPL photolysis alone, even under optimized conditions. Therefore, enantioselective synthesis as possible pathway for enantioenriched extraterrestrial amino acids caused by UVCPL was proposed (see Chapter 4). However also here, the degree of inducible ee values is limited based on the specific anisotropy factor (see Chapter 3). A second hypothesis suggests reiterated asymmetric photolysis of already photolyzed amino acids, which would yield increased ee values, but would also lead to an almost complete destruction of the produced enantiomerically enriched material [73]. In a third hypothesis, 
an enantiomerically enriched catalyst formed by asymmetric photolysis is acting as promoter in the generation of amino acids which would lead to substantial enantiomeric excess in the products.

In general, it could be observed that the CM meteorites, Murchison and Murray, have nearly identical amino acid distributions [85]. The same finding could be observed for the distribution of the most abundant amino acids in Orgueil and Ivuna-two CI carbonaceous chondrites. However, the distribution in the latter differs from the distribution found in the CM meteorites. The striking difference in the amino acid composition of these carbonaceous chondrite classes suggests that they originate from a different type of parent body, possibly an extinct comet [86]. It should be noted that similar distribution patterns of amino acids from different meteorites have to be carefully weighted, since meteorites themselves are characterized by significantly quantitative heterogeneity of their chemical distribution [54,65].

Figure 2. Diamino acid enantiomers (highlighted in red and green, respectively) detected in a sample of the Murchison meteorite. A hot water extract of the meteorite was hydrolyzed with $6 \mathrm{M} \mathrm{HCl}$, derivatized to form ethoxycarbonyl diamino acid ethylester [92], separated by enantioselective capillary gas chromatography on a Chirasil-D-Val stationary phase, and detected by a mass spectrometer at 175 a.m.u. The insets show the external standards of the separated diamino acids D,L-2,3-diaminopropanoic acid (DAP) and D,L-2,4-diaminobutyric acid (DAB) enantiomers (Std) detected in the total ion current, and a serpentin blank (Blk) detected in the single ion monitoring mode of mass trace 175 a.m.u., that had been heated before extraction for $4 \mathrm{~h}$ at $500{ }^{\circ} \mathrm{C}$ and passed through the analytical protocol; a.m.u., atomic mass units [69].

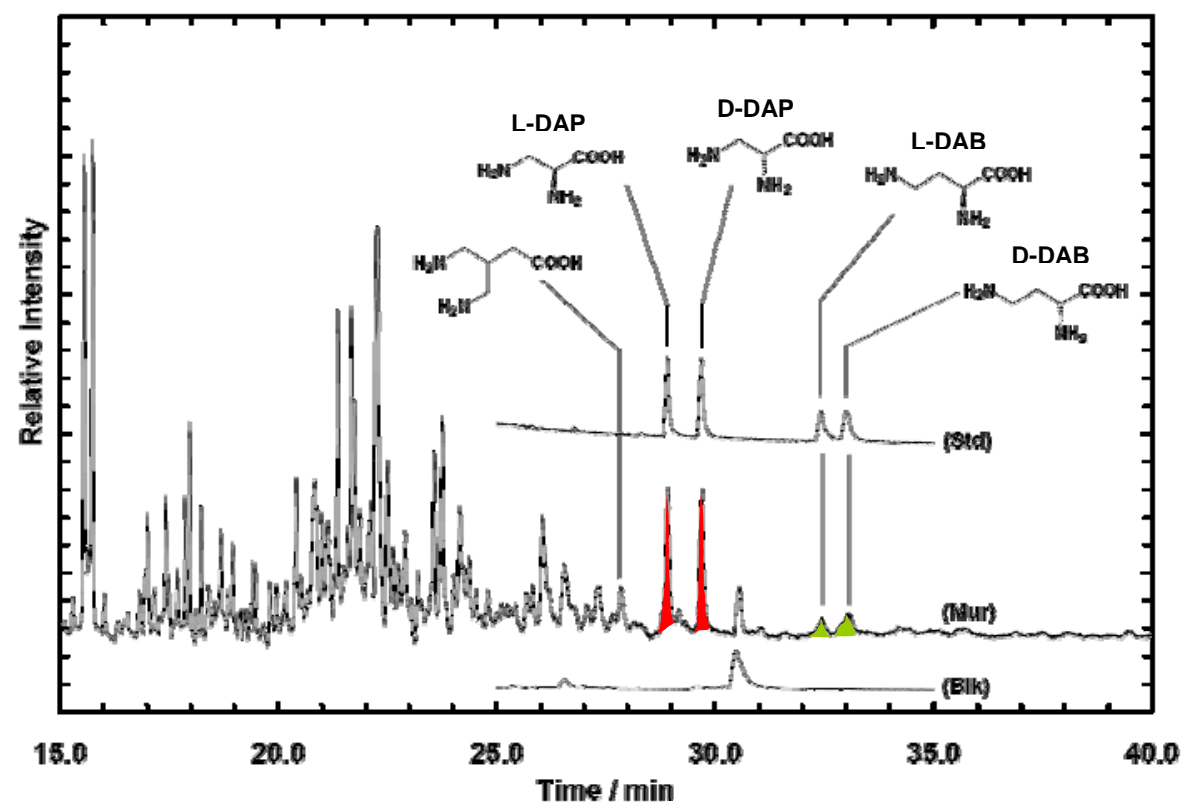

A unique finding was reported by Meierhenrich et al. [69] who identified five diamino monocarboxylic acids (diamino acids) in the Murchison meteorite (Figure 2). This is of considerable relevance because diamino acids have been proposed as backbone building blocks of the so-called peptide nucleic acids (PNAs) [87]. PNA, on the other hand, has been suggested as a possible pre-RNA genetic material, in which a peptide chain takes over the role of the sugar-phosphate 
backbone (Figure 3) [88]. This makes it all the more astonishing as Nielsen proposed a PNA based on 2,4-diaminobutanoic acid, among others, which was one of the diamino acids which could actually be identified in the Murchison meteorite by the team of Meierhenrich.

Figure 3. Peptide nucleic acid (PNA) and ribonucleic acid (RNA) structures. In general, one distinguishes between daPNA composed of diamino acids (da) and aegPNA, in which the molecular backbone is constructed by $\mathrm{N}$-aminoethylglycine (aeg). The structure given below depicts daPNA using the diamino acid 2,4-diaminobutyric acid (DAB) in its molecular backbone. Racemic DAB was identified in the Murchison meteorite (Figure 2).

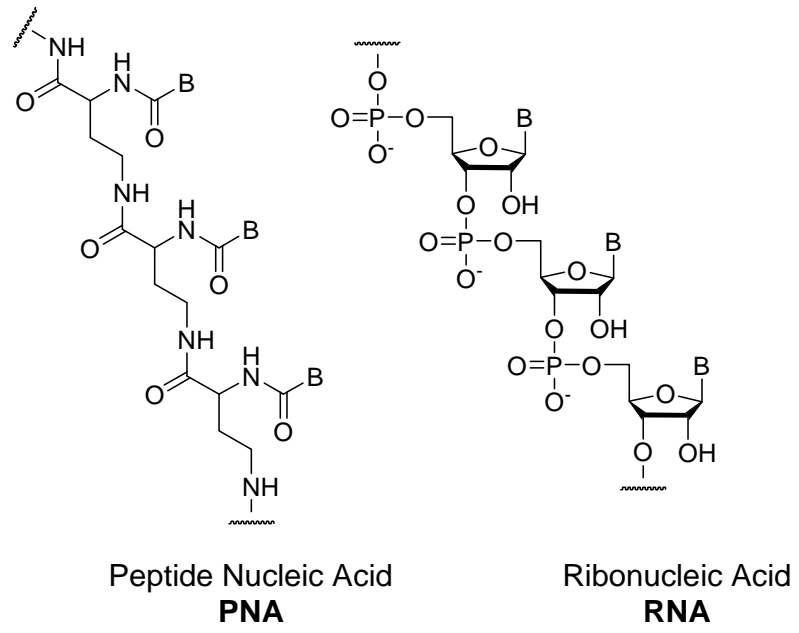

Enantioenriched meteoritic $\alpha$-amino acids also became important in another prebiotic scenario: The poor yields of the prebiotic synthesis of ribose in the formose reaction have often been cited as the reason why prebiotic synthesis of nucleotides was unlikely. Pizzarello and Weber, however, have recently demonstrated that the non-racemic amino acids in meteorites such as isovaline can transfer their chiral information to other prebiotic building blocks, for example in the formation of tetrose sugars from glycolaldehyde by aldol addition [71]. Similar stereoselectively catalytical properties could also be observed for asymmetric amino acid dipeptides in the formation of pentose and tetrose sugars $[89,90]$. In this context it is also interesting to note that $\alpha$-methyl-amino acids, such as isovaline, are known as "long-term carriers" of chiral information because of their relative stability to chemical racemization [91], whereas sugars and other amino acids missing substituents at the "weakly" acidic stereogenic C2 position could have lost any enantiomeric excess, if it existed, via racemization. It is therefore believed, that the $\alpha$-methyl amino acids, the most abundant non-racemic amino acids in carbonaceous chondrites, in particular isovaline with the highest ee value measured so far, might have acted as chiral catalysts during chemical evolution on early Earth.

\subsection{Reaction Mechanisms for the Formation of Interstellar Amino Acids}

An important and much discussed astrophysical question concerns the mechanism leading to the formation of interstellar amino acids. Multiple pathways are suggested for the amino acids detected in carbonaceous chondrites and those produced in interstellar ice analogs. 
Among them, the most discussed pathway is the Strecker-type mechanism (Strecker-cyanohydrin), which is assumed to occur during aqueous alteration within meteoritic parent bodies [25]. This Strecker-type reaction involves the formation of aminonitriles from hydrogen cyanide (HCN), ammonia and carbonyl compounds such as aldehydes and ketones, which would lead to the formation of amino acids by subsequent hydrolysis [93-95]. A variety of aldehydes and ketones are known to be present in the Murchison meteorite, which supports this reaction pathway as well as the high abundances of aminoisobutyric acid and isovaline found in this chondrite which will preferentially be formed under these conditions. However, $\beta-, \gamma-$, and, $\delta$ - amino acids as well as diamino acids identified after acid hydrolysis in both carbonaceous chondrites and interstellar ice analogs, cannot be generated by the Strecker-cyanohydrin pathway, demanding for an alternative synthetic pathway.

Another interesting finding in this context is the fact that ammonia, $\mathrm{HCN}$, formaldehyde as well as cyanoacetylene, one of the potential precursors for $\beta$-alanine, present in high amounts in $\mathrm{CM}$ meteorites, have been detected also in the coma of comet Hale-Bopp [96]. However, ketones and aldehydes, necessary precursors for the postulated Strecker pathway could not been found. Furthermore, several models exclude the presence of liquid water in the interior of comets, required for aqueous alteration and the Strecker-type mechanism [97]. Moreover, compound-specific isotopic analyses of amino acids found in the Murchison meteorite revealed that various isomeric subgroups of these compounds differ in ${ }^{13} \mathrm{C}$ fractionation trends [71]. Whereas $\delta \mathrm{D}$ values obtained from compound-specific D/H measurements [82], on the other hand, do not vary between but rather within the subgroups, suggesting diverse synthetic mechanisms of interstellar amino acids.

Other potential chemical pathways include specific radical-radical mechanisms, modified radical-radical mechanisms with nitriles as amino acid precursor molecules [57], and ion-molecule reactions in the gas-phase, which could be studied both in the laboratory and theoretically [98,99], as well as such reactions during molecular cloud collapse [100]. The isotopic enrichment in the meteoritic amino acids, particularly in deuterium, indicates ion-molecule reactions at very low temperatures like they occur in cold molecular clouds [101]. Finally, the formation of amino acid structures in interstellar ice analogs also suggests a photochemical reaction pathway in the interstellar medium, rather than the production in aqueous solution on interstellar parent bodies $[34,35]$.

In conclusion, predicting the "correct" reaction mechanisms leading to the formation of interstellar amino acids may provide important information on the degree of truly interstellar organic species survived during the solar nebula condensation phase.

\section{Asymmetric Photolysis of Racemic Organic Molecules}

Already in 1929, asymmetric photolysis of organic molecules with circularly polarized light in ultraviolet wavelengths (UVCPL) was shown to yield optically active products by enantioselective photodecomposition of ethyl- $\alpha$-bromopropionate and $N, N$-dimethyl- $\alpha$-azidopropionamide [19-21]. In 1974, irradiation of camphor yielded the highest reported photochemically induced ee value (20\%) with a $99 \%$ photodecomposition rate [73].

Note that CPL-mediated experiments depend on circular dichroism of the reactant $\left(\Delta \varepsilon=\varepsilon_{\mathrm{RCPL}}-\varepsilon_{\mathrm{LCPL}}\right)$, i.e., on the preferential photodestruction of one enantiomer having the higher absorption coefficient $(\varepsilon)$. The efficiency by which an enantioselective photolysis can be 
achieved depends on the so-called anisotropy factor $g(g=\Delta \varepsilon / \varepsilon)$ and the extent of the reaction (photodecomposition) [73]. Within the protoeinaceous amino acids, leucine was described to possess the highest anisotropy factor ( $g=0.0244$, in $0.1 \mathrm{M} \mathrm{HCl}$ solution) [102] and therefore has preferentially been studied in enantioselective photolysis experiments. It should be noted that the optical properties ( $g_{\max }$ values) of amino acids in solution significantly depend on the $\mathrm{pH}$ value [74]. The highest magnitude of the $g$ factors $(0.025-0.035)$ among the proteinogenic amino acids were found for the aliphatic (alanine, leucine, valine, isoleucine, and proline) and two hydroxyl amino acids (serine and threonine), respectively, at $\mathrm{pH} 1$.

However, Flores et al. [103] reported an enantiomeric excess for originally racemic leucine in acidic solution of $2.50 \%$ ( $l$-CPL, left-handed) and $1.98 \%$ ( $r$-CPL, right-handed) using UVCPL at $212 \mathrm{~nm}$, with photolysis rates of $75 \%$ and $59 \%$, respectively.

Further, extensive attempts of inducing higher ee into chiral molecules have been made in strong acidic solutions at (a) different $\mathrm{pH}$ values [74,104], (b) the use of two photon excitation processes [105], and (c) with elliptically polarized light [106], yielding enantiomeric excess $e e_{\mathrm{L}}$ values of (a, b) $0.2-0.7 \%$ and (c) 3\%, respectively, for D,L-leucine. All of these experiments were carried out in aqueous solution, where the optical activity of amino acids arises from the $\left(\pi^{*}, n\right)$ electronic transition of the carboxylic group (chromophore) bound to their $\alpha$-carbon. Excitations of higher energetic electronic transitions are impossible due to the water absorbing below $200 \mathrm{~nm}$.

Surprisingly, most of the CD spectra of amino acids are also recorded in liquid phase, where the $\mathrm{pH}$ value of the medium by protonating and deprotonating the acid and basic groups as well as the nature of the solvent modify the optical properties [107] and thus signs and intensities of CD bands. These problems will be avoided when recording CD spectra of amorphous solid-state films which are considered to be more representative for interstellar conditions [7,108]. Moreover, in the extraterrestrial scenario of biomolecular asymmetry early-type stars would have emitted UV light with wavelength below $200 \mathrm{~nm}$ [28]. Therefore, studying photochemical interactions of amino acids with left- and righthanded CPL in the solid state might become of particular interest in the future. Figure 4 represents one of the first circular dichroism spectra of L-leucine in its solid state recorded down to $130 \mathrm{~nm}$.

In 2005, first results on the irradiation of racemic leucine in the micro-cristalline-state using circularly polarized synchrotron radiation (CPSR) were reported [108]. After 70\% photodecomposition, an enantiomeric excess of $+2.6 \%$ in D-leucine irradiated with $r$-CPSR at $6.8 \mathrm{eV}(182 \mathrm{~nm})$ could be identified using enantioselective gas chromatography. In a follow-up experiment the same authors could obtain an even higher ee of 5.2\% in D-leucine after irradiating racemic leucine in the solid-state with $r$-CPSR for 55h (Figure 5). However, this experiment could not be verified by an oppositely irradiated sample with $l$-CPSR. The quantities in that sample were found to be insufficient to precisely measure the ee due to the high recorded photolysis rate (99.23\%) of that sample [109].

This experimental design successfully demonstrated that an enantiomeric excess using VUV-CPSR and amino acids in their solid-state-representing more realistic interstellar conditions-is feasible. However, this actual record of a photochemical-induced ee into amino acids should be an incentive for others to reach even higher values, probably by studying $\alpha$-methyl amino acids such as isovaline, the highest enantiomerically enriched amino acid found in meteorites. These experiments might well explain the processes leading to the interstellar asymmetric formation of biomolecular precursors. 
Figure 4. Circular dichroism spectra* of solid state L-leucine in the VUV range (130-330 nm) measured from an amorphous film of sublimated amino acid (750 nm-thick). The sample was rotated around the beam axis in steps of $90^{\circ}$ in order to minimize the contributions of VUV birefringence and linear dichroism to the signal. (* recorded at the synchrotron facility ISA, Aarhus University, Denmark) [109].

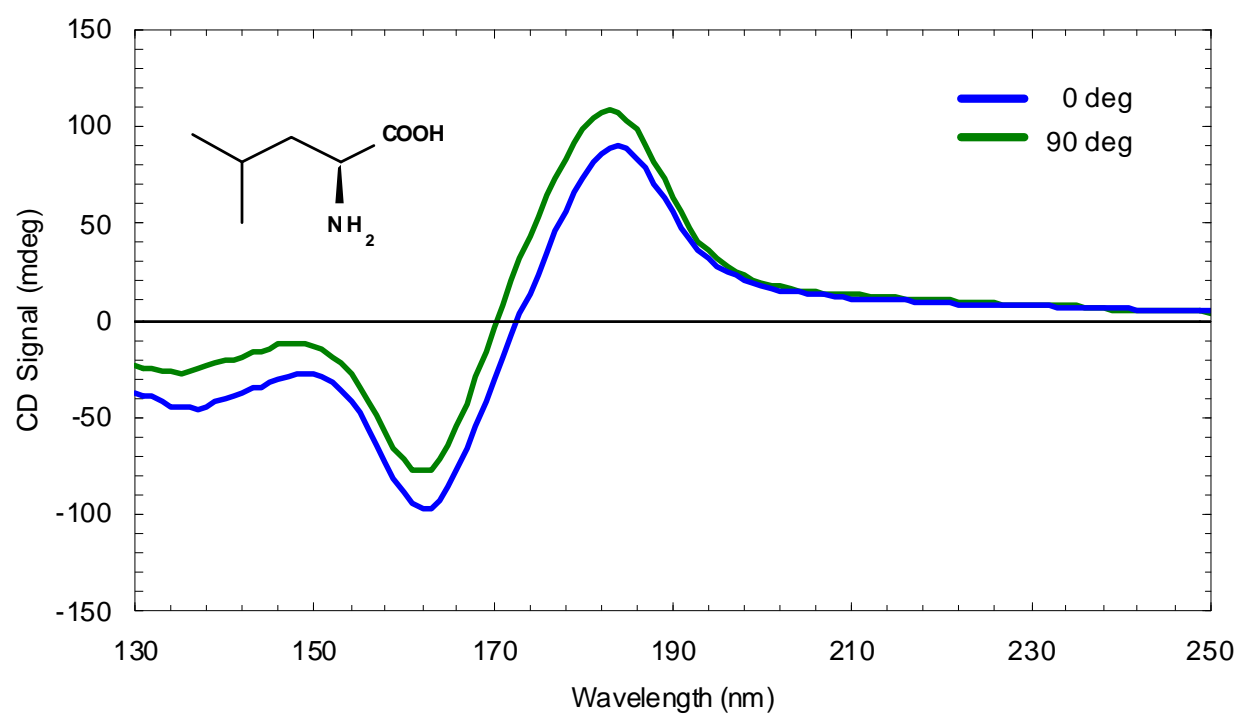

Figure 5. Gas chromatographic enantiomer separation of D, L-leucine. The black line represents a sample irradiated under right-circularly polarized VUV light at the DESIRS beamline (Synchrotron SOLEIL, Paris), the red one corresponds to a non-irradiated commercial sample of racemic D, L-leucine. Enantiomer separation of L- and D-leucine is achieved on a Chirasil-L-Val under the following operating conditions: constant He flow, $1.3 \mathrm{~mL} / \mathrm{min}$; oven temperature program, $70{ }^{\circ} \mathrm{C}$ to $130{ }^{\circ} \mathrm{C}\left(10{ }^{\circ} \mathrm{C} / \mathrm{min}\right.$ rate $), 130{ }^{\circ} \mathrm{C}$ to $180{ }^{\circ} \mathrm{C}\left(2{ }^{\circ} \mathrm{C} / \mathrm{min}\right.$ rate $)$, finally held isothermal for $10 \mathrm{~min}$; detection mode, SIM $158 \mathrm{~m} / z$.

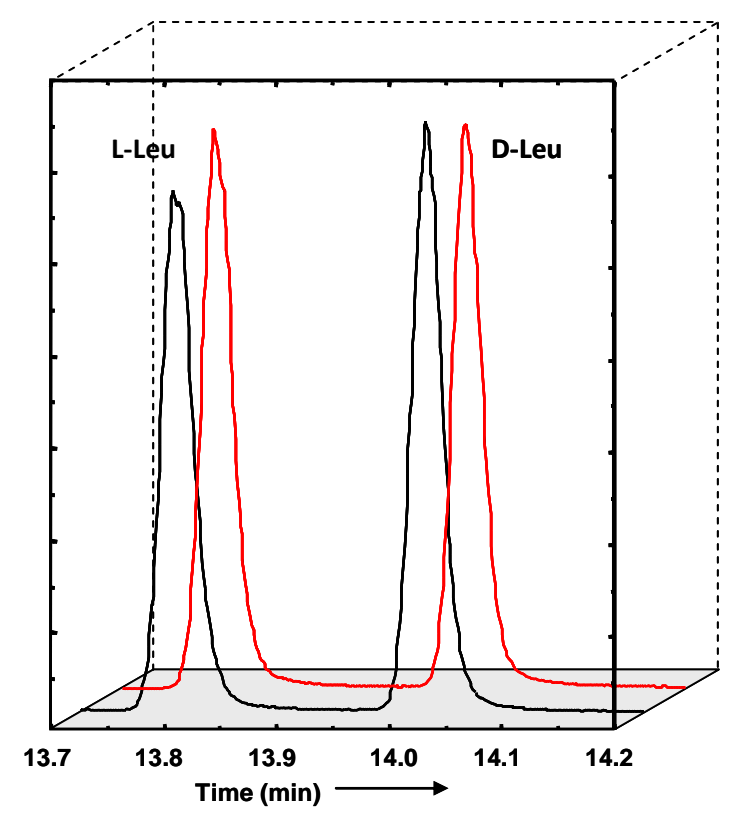




\section{Enantioselective Photosynthesis}

Circularly polarized light was shown to be capable of inducing asymmetric photochemical reactions in organic molecules as highlighted above (Chapter 3). It was therefore assumed that CPL could also be effectuating enantioselective syntheses starting with achiral reactants. The first experiment was published by Kagan et al. [110] who asymmetrically synthesized octa- and nonahelicene with optical purities below $2 \%$. These results could indeed confirm the so called "Curie principle", meaning that circular or elliptically polarized light itself, has a true chirality [15,16,102,111] and thus, photosynthesis induced by such radiation sources will lead to non-racemic products.

Since racemic amino acid structures could have been formed under interstellar conditions using unpolarized light ([34,35]; see also Chapter 2.1), the next logical step would be the use of similar conditions but circular polarized light to directly generate enantioenriched amino acids.

Very recently, preliminary results for this hypothesis were demonstrated by two different research groups. Takano et al. [112] first prepared complex organic compounds by irradiating a gaseous mixture of carbon monoxide, ammonia, and water-molecules which are actually present in the ISM-with 3.0 MeV protons. The synthesized precursor molecules were then irradiated in the liquid phase with left- and right-circularly polarized synchrotron light $(\lambda>200 \mathrm{~nm})$. After the hydrolysis of the residues several amino acids could be detected using reversed-phase liquid chromatography; for example, glycine, alanine, serine, aspartic acid, $\alpha$-aminobutyric acid, valine, and $\beta$-alanine. For one chiral amino acid-alanine-an enantiomeric excess of $+0.44 \%$ by $r$-CPSR and $-0.65 \%$ by $l$-CPSR was reported with a standard deviation of \pm 0.31 and $\pm 0.23(1 \sigma)$, respectively.

A second irradiation experiment of interstellar ice analogs with VUV-CPSR was reported by Nuevo et al. [113,114]. The two interstellar gaseous mixtures containing water, methanol, and ammonia were directly irradiated with either left- or right-circularly polarized synchrotron light, for $56 \mathrm{~h}$ and $43 \mathrm{~h}$, respectively. The energy of the CPSR $(7.45 \mathrm{eV} ; 167 \mathrm{~nm})$ used for this experiment corresponds to a maximum of $r$-CPSR and l-CPSR, respectively, in the CD spectrum of several amino acids. Carbon-13 isotopically labeled reactants in the initial gaseous mixture were used to exclude any biological contamination.

Among several organic compounds, eight amino acids were identified in the acid hydrolyzed residue by enantioselective gas chromatography: sarcosine, alanine, glycine, aminobutyric acid, $\beta$-alanine, aspartic acid, diaminopropanoic acid (DAP), and 3,3'-diaminoisobutyric acid (Figure 6). Enantiomeric excesses were measured for alanine and DAP, the most abundant proteinaceous and nonproteinaceous chiral amino acids. The ee values were found to be in the order of $1 \%$, however, with inconclusive error bars $( \pm 1 \%, 3 \sigma)$.

Even if the small ee values found for alanine in this kind of experiment seems to be disappointing at first sight, they actually match with the L-enantiomeric excess measured for alanine in the Murchison meteorite of 1.2\% [115]. Moreover, for the first time, the generation of amino acids and diamino acids by irradiating an interstellar ice mixture exclusively by circularly polarized light was successfully demonstrated.

In 2009, Takahashi et al. [116] measured the optical anisotropy of different racemic amino acids in their solid-state before and after irradiation with circularly polarized light. The authors could successfully introduce an enantiomeric excess into the originally racemic films expressed as CD signal. 
Figure 6. Gas chromatograms showing the enantiomeric distribution of alanine and 2,3-diaminopropanoic acid (DAP) in simulated interstellar ices photoprocessed under linear (red) or right circularly polarized (blue) VUV light at the SU5 beamline (Synchrotron Lure, Orsay, France). Enantiomer separations were achieved using enantiselective capillary gas chromatography on a Chirasil-D-Val stationary phase.

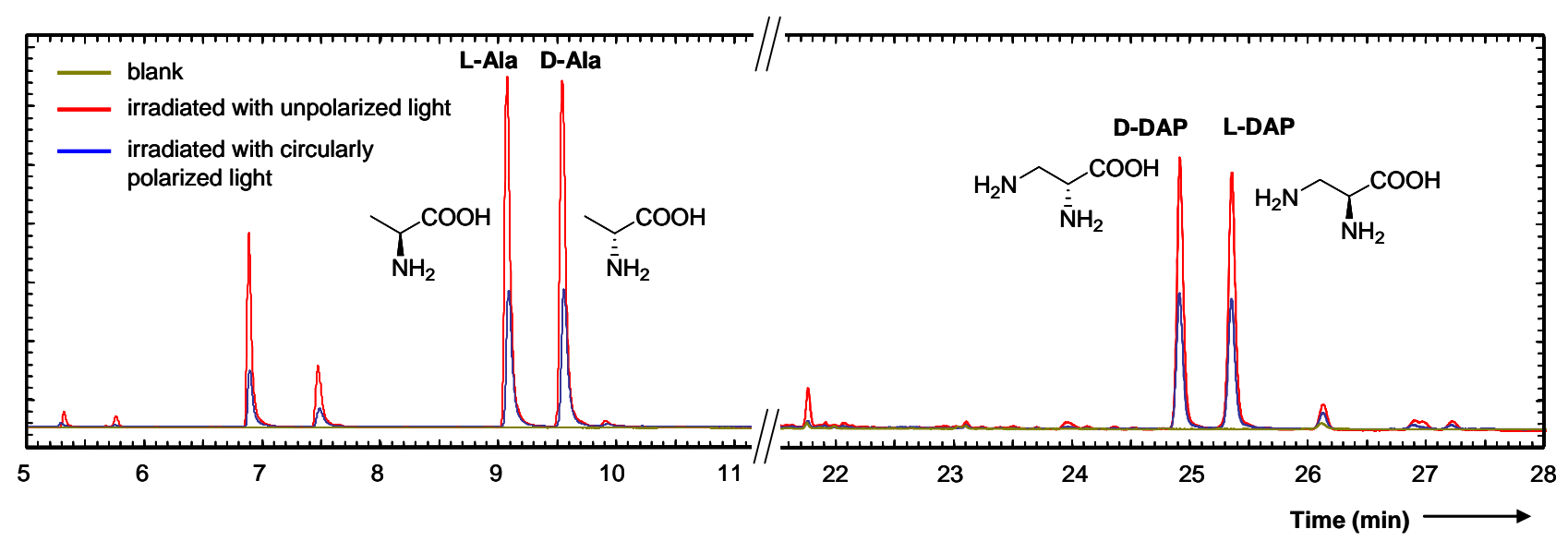

Future results in these highly sophisticated experiments using interstellar ice analogs, high-flux synchrotron circularly polarized radiation, enantioselective separation techniques, and circular dichroism spectrometers are decidedly important to explain whether circularly polarized light might have directly effectuated the asymmetric synthesis of amino acids found in meteorites.

\section{Rosetta-The First Landing on a Cometary Nucleus}

The early Earth provided suitable conditions for the evolution of life, but lacked organic reduced material necessary for the origin of life. On the contrary, outer solar system bodies are rich in water and organic material, and there exists abundant evidence on the organic input from micrometeorits, asteroids, and comets on Earth surface [4]. Even now, hundreds of tons of dust and meteorites from these bodies reach our planet every day [117]. Therefore, it is believed today that the organic molecules observed in comets may be a source of prebiotic molecules that led to the origin of life on Earth. Thus, the in situ analysis of comets, in particularly of their nucleus will offer us new insights into life's beginning.

Comets are agglomerates of icy grains, which were created in the outer solar system-far away from the Sun-without being integrated into planets and therefore considered to be the most pristine interstellar bodies [45]. Recent space missions to the long-period comets Halley, or IR observations of Hyakutake and Hale-Bopp allowed to have a closer look on their inventory: About $30 \%$ of the cometary grains are dominated by light elements $\mathrm{C}, \mathrm{H}, \mathrm{O}$, and $\mathrm{N}$, comparable to carbonaceous chondrites. Chemical compounds of exobiological interest identified in the coma of comets are: $\mathrm{HCN}$, $\mathrm{H}_{2} \mathrm{CO}, \mathrm{NH}_{3}, \mathrm{CH}_{4}, \mathrm{HCOOH}$, acetylene $\left(\mathrm{C}_{2} \mathrm{H}_{2}\right)$, acetonitrile $\left(\mathrm{CH}_{3} \mathrm{CN}\right)$, hydrogen isocyanide (HNC), isocyanic acid $(\mathrm{HNCO})$, cyanoacetylene $\left(\mathrm{HC}_{3} \mathrm{~N}\right)$, and thioformaldehyde $\left(\mathrm{H}_{2} \mathrm{CS}\right)$ (for a review, see [42]). Most of the neutral cometary molecules are also identified in the gas phase of the ISM [46,118] indicating a relationship between comets and interstellar clouds. There is a broad agreement that 
organic molecules detected in the coma originate from the dust that must have existed in the solar nebula at the very time and place of comet formation [37,119].

Until today, only the molecular composition of comet Halley's coma-ejected when the comet comes close to the sun and frozen gases from the surface layers of the nucleus sublimate and leave the marginal gravity of the comet with some dust particles-could be observed by UV-, IR-, and mass-spectroscopy. Nevertheless, based on these findings the astrobiological community has good reasons to assume that specific molecular compounds including chiral molecules, such as amino acids, or at least their precursor molecules, can be present in cometary matter.

Indeed, there will be a rendezvous with a comet in the near future: As part of the ROSETTA mission, the PHILAE Lander was launched on-board the European Space Agency (ESA) ROSETTA spacecraft. In November 2014, PHILAE will be released from the orbiter and-hopefully-soft-land on the nucleus of Comet $67 \mathrm{P} /$ Churyumov-Gerasimenko to bring the laboratory equipment to the comet rather than pick-up the sample to take-away. For the first time the study of enantiomeric excesses in organic molecules on an extraterrestrial body is part of the scientific objectives.

Therefore, the Cometary Sampling and Composition Experiment (COSAC) was designed to analyze cometary surface and sub-surface samples in order to separate, identify, and quantify chiral and achiral molecules [120]. The in situ separation of chiral compounds will be performed by enantioselective gas chromatography [121] combined with a TOF mass spectrometer. The detection of a L-enantiomeric excess in cometary molecules, should it be present, would support the hypothesis that extraterrestrial amino acids have predetermined life's left-handedness of our proteinaceous amino acids.

Another important investigation will focus on two marker compounds: polyoxymethylene (POM) and hexamethylenetetramine (HMT), both found in the residues of interstellar ice analogs $[58,122,123]$. Their presence or absence could indicate some of the predominant processes in the comet's ice history. Accordingly, POM is a good indicator for thermal processes while HMT indicates UV photolysis and proton irradiation processes [60].

\section{Criticism}

Critics on the theory of an extraterrestrial photochemical origin of enantiomerically enriched amino acids have been raised since the beginning.

(1) The first demand is that amino acids and/or their precursor molecules have to be formed in the interstellar medium: Glycine, the simplest of our proteinogenic amino acids, has been claimed to be detected in (hot) molecular cores [124]. Unfortunately, in a detailed analysis of the evidence, glycine lines which should have appeared were not found and this claim has not been confirmed [125]. It should be noted, that amino acids can easily be photo-destroyed in UV-rich space environments even by long wavelength $(>200 \mathrm{~nm}$ ) photons [126] and therefore should preferentially be present in shielded space regions like in icy grains. Aminonitriles-precursor molecules of amino acids-however were found to be more resistant to photochemically destruction in this study.

However, in 2009, extraterrestrial glycine was directly detected in an authentic cometary sample, returned to Earth by the Stardust NASA mission, using gas chromatography-mass spectrometry coupled with isotope ratio mass spectrometry [127]. Further lines of evidence for the existence of 
extraterrestrial amino acids are the successful laboratory production of amino acids by simulating interstellar conditions [34,35] and their identification in meteorites [54].

(2) Secondly, UV CPL must be present in space and favor the L-enantiomer for all the primordial chiral amino acids involved in the genesis of life on Earth. In a first proposed scenario for interstellar UV CPL, a molecular cloud in the vicinity of a supernova explosion is exposed to ultraviolet radiation from its central neutron star, and the racemic molecules of the organic mantle of the interstellar grains could be asymmetrically photolyzed [26,27,38]. The more likely source of CPL was presented by Bailey and coworkers $[28,29,128]$ based on the observation of a large degree of circular polarization ( -5 to $+17 \%)$ in IR wavelengths in the Orion OMC-1 star formation region. It is believed that CPL is produced by Mie scattering on non-spherical grains aligned by a magnetic field, multiple scattering, or dichroism involving multiple clouds or twisted magnetic field lines [29,129]. Nevertheless, no direct observation of CPL in the UV range could be carried out because of the dust extinction in these regions. So far, only model calculations indicate similar circular polarization rates for the UV domain as observed in the IR $[28,130]$.

Moreover, most efficient asymmetric photolysis of biogenic amino acids requires excitation by distinct bands of the CPL spectrum to overcome the problem that CD bands sum to zero over the entire spectral range [131] (Kuhn-Condon zero sum rule). However, the wavelength integral of the CD index weighted by the actual broad-band CPL spectrum in star formation regions [28] yields a non-zero effective CD coefficient $\Delta \varepsilon$. According to Cerf and Jorissen [133] the resulting net enantioselective effect must give an excess of L-enantiomers for all proteinogenic amino acids [132]. These authors argued against the postulated asymmetric photolysis scenario since amino acids like tryptophan and proline violate this condition due to more complex CD spectra in the 200-230 nm wavelength region compared to alanine, valine and leucine. Takahashi et al. [109] recently reported intense CD transitions below $200 \mathrm{~nm}$ using a commercial CD photospectrometer. This spectacular finding has to be included in the discussion of how extraterrestrial broad-band CPL can lead to enantioselection since circular polarization of interstellar radiation is probably expanded into the vacuum UV spectral region. In this respect, asymmetric photolysis studies of (i) solid state proteinogenic amino acids using (ii) broad-band VUVCPL rather than monochromatic light with (iii) wavelength below $200 \mathrm{~nm}$ matching as closely as possible the conditions prevailing in space would be of great interest.

Another critical point commonly raised is that a significant enantiomeric excess requires a high photolysis rate [73], e.g., to reach an amino acid ee of up to $10 \%$, photodestruction of at least $99.99 \%$ would be necessary. Moreover, to keep any introduced enantiomeric enrichment, UV-CPL should have been the last effective irradiation seen by the biomolecular precursor molecules. Otherwise, this a priori small and hard reached enantiomeric asymmetry could have been easily degraded again thereafter [126]. However, if we assume that the L-enantiomeric excess in the meteoritic $\alpha$-methyl amino acids [22-24,71] was a general feature of the solar nebula, it could have been implemented in the Solar System itself leading to our next requirement.

(3) The non-racemic amino acids must be successfully "shipped" to the early Earth: different studies indicate that the transport from outer space to the prebiotic Earth was possible either by direct accretion of the interstellar dust $[39,42]$ or through impacts of meteorites, comets, and interstellar dust particles (IDPs, $\sim 100 \mu \mathrm{m}$ radius) $[4,40,41]$. It is assumed, for example, that the ice mantles of the submicron dust grains in IDPs and in comets [95] as well as refractory organic matter (ROM) in 
carbonaceous chondrites [134] acted as a shield for the organic chiral molecules protecting them from destructive processes. The presence of ROM in meteorites is also discussed to not only preserve biochemically important species but also, and above all, avoid racemization of enantioenriched precursors in such a complex organic network. Indeed, some organic matter including amino acids could survive this harsh trip without being destroyed when passing the atmosphere and striking the Earth's surface, as shown by a wide variety of identified organic compounds in fallen meteoritic samples such as Murchison [54,55,65].

Meteorites represent only a tiny fraction of the total material delivered to Earth from space [117]. The largest input would have been from interplanetary dust particles. Accordingly, interstellar dust collections in Greenland and Antarctica ice sheets [135] show that the Earth is still soaking up interplanetary dust as micrometeorites. However, only one amino acid, $\alpha$-aminoisobutyric acid, could be identified in one of these Antarctic micrometeorites so far [136,137]. Even if we do not consider meteorites as the main supplier for enantioenriched amino acids to the early Earth, they in fact demonstrate their existence in outer space and leave the door open for further discoveries of any enantiomeric excess in other interstellar bodies as well.

(4) Since the enantiomeric enrichment initiated by CPL is small and may be further degraded by racemization, amplification mechanisms would come into play for increasing this enantiomeric excess. Therefore, cycles of partial polymerization and hydrolysis, evaporation and crystallization, as well as kinetic resolution have been proposed to increase the enantiomeric excess from very few percent towards homochirality (reviewed in [1]). Most promising seems to be the asymmetric amplification using enantioenriched auxiliaries with either catalytic [138] or autocatalytic function (Soai reaction type) [139-141].

(5) The last critical point deals with the precise determination of any small enantiomeric excess using gas or liquid chromatography. In principle, two different enantioselective separation methods can be applied to measure ee of D, L-amino acids: either derivatization with an achiral reagent followed by the separation of the enantiomers on a chiral stationary phase or via derivatization with a chiral agent and separation of diastereomers on an achiral stationary phase. The latter requires several conditions for its use in accurate ee determination investigations including quantitative enantiomeric purity of the derivatization auxiliary, complete absence of racemization of both reactants during derivatization, and identical reaction kinetics of both enantiomers with the chiral agents. Therefore, the use of the achiral derivatization approach combined with the separation on a chiral stationary phase should be applied preferably due to the absence of the above mentioned factors [142].

In general, any small enantiomeric excess obtained should carefully be discussed regarding resolution $(R)$ of closely eluting enantiomers ( $R$ values should be given), applied derivatization method, and reverse elution order of enantiomers by applying the oppositely configured chiral stationary phase to verify the analytical results.

\section{Conclusions}

We have outlined that asymmetric photoreactions are capable of producing slight enantiomeric enrichments in racemic mixtures of organic molecules. The identification of several non-racemic $\alpha$-amino acids in different meteorites supports the theory of an extraterrestrial origin of biomolecular 
asymmetry. The synthesis of non-racemic DAP in interstellar ice analogs using UV-CPL and its presence in meteoritic samples give good reasons to believe that diamino acids significantly contributed to the origin of life's genetic material.

Future research will certainly continue studying asymmetric photolysis and enantioselective photosynthetic reaction pathways leading to enantioenriched organic molecules. These molecules must not necessarily be amino acids. But amino acids attracted the interest of the research community since (a) they were identified in carbonaceous meteorites and in simulated interstellar ices, (b) amino acids are required for the biosynthesis of stereoregular proteins, and (c) diamino acids such as DAP-suggested for the PNA backbone-may have been the only diamino acids needed for the origin of life. In this spirit, fine stereoselective-interactions between biomolecules of different kinds of molecular families are a remarkable feature of present life and should be included in the scientific discussion on the origin of biomolecular asymmetry.

The understanding of how these first chiral molecules became assembled into living systems has become a topical research field, in which proteins are used as catalysts and various oligonucleotides as information storage molecules. Scientific studies on the origins of life in general and on the origin of biomolecular homochirality in particular are of interdisciplinary interest and will continue producing important and highly fascinating data on our origins.

\section{Acknowledgements}

This work was funded by the ANR-07-BLAN-0293, the SOLEIL synchrotron 20060143, 20080058, 20080405, 20090297, the Institute for Storage Ring Facilities at Aarhus University ISA-09-102 and the European Community's Integrated Infrastructure Initiative Activity on Synchrotron and Free Electron Laser Science, contract number RII3-CT-2004-506008 and the Seventh Framework Program (FP7/2007-2013) under grant agreement n ${ }^{\circ} 226716$.

\section{References and Notes}

1. Bonner, W.A. The origin and amplification of biomolecular chirality. Orig. Life Evol. Biosph. 1991, 21, 59-111.

2. Miller, S.L. A production of amino acids under possible primitive earth conditions. Science 1953, $117,528-529$.

3. Sagan, C.; Khare, B.N. Long-wavelength ultraviolet photoproduction of amino acids on the primitive Earth. Science 1971, 173, 417-420.

4. Chyba, C.F.; Thomas, P.J.; Brookshaw, L.; Sagan, C. Cometary delivery of organic molecules to the early earth. Science 1990, 249, 366-373.

5. Kasting, J.F.; Catling, D. Evolution of a habitable planet. Annu. Rev. Astron. Astrophys. 2003, 41, 429-463.

6. Stribling, R.; Miller, S.L. Energy yields for hydrogen cyanide and formaldehyde syntheses: The hydrogen cyanide and amino acid concentrations in the primitive ocean. Orig. Life Evol. Biosph. 1987, 17, 261-273.

7. Meierhenrich, U. Amino Acids and the Asymmetry of Life; Springer-Verlag: Berlin-Heidelberg, Germany, 2008; p. 241. 
8. Karagunes, G.; Coumoulos, G. A new method of resolving a racemic compound. Nature 1938, $142,162-163$.

9. Bonner, W.A.; Kavasmaneck, P.R.; Martin, F.S.; Flores, J.J. Asymmetric adsorption of alanine by quartz. Science 1974, 186, 143-144.

10. Bonner, W.A. The quest for chirality. AIP Conf. Proc. 1996, 379, 17-49.

11. Yamagata, Y. A hypothesis for the asymmetric appearance of biomolecules on earth. J. Theor. Biol. 1966, 11, 495-498.

12. Vester, F.; Ulbricht, T.L.V.; Krauch, H. Optical activity and parity violation in ß-decay. Naturwissenschaften 1959, 46, 68.

13. Goldhaber, M.; Grodzins, L.; Sunyar, A.W. Evidence for circular polarization of bremsstrahlung produced by ß-rays. Phys. Rev. 1957, 106, 826-828.

14. Bonner, W.A. Experiments on the origin of molecular chirality by parity nonconservation during ß-decay. J. Mol. Evol. 1974, 4, 23-39.

15. Barron, L.D. Fundamental symmetry aspects of chirality. BioSystems 1987, 20, 7-14.

16. Barron, L.D. Can a magnetic field induce absolute asymmetric synthesis? Science 1994, 266, 1491-1492.

17. Rhodes, W.; Dougherty, R.C. Effects of electric and magnetic fields on prochiral chemical reactions: Macroscopic electric and magnetic fields can cause asymmetric synthesis. J. Am. Chem. Soc. 1978, 100, 6247-6248.

18. Rikken, G.L.J.A.; Raupach, E. Pure and cascaded magnetochiral anisotropy in optical absorption. Phys. Rev. E Stat. Phys. Plasmas Fluids Relat. Interdiscip. Topics 1998, 58, 5081-5084.

19. Kuhn, W.; Braun, E. Photochemical preparation of optically active substances. Naturwissenschaften 1929, 17, 227-228.

20. Kuhn, W.; Knopf, E. The preparation of optically active compounds by the aid of light. Z. Physik. Chem. 1930, 7, 292-310.

21. Kuhn, W.; Knopf, E. Photochemical preparation of optically active substances. Naturwissenschaften 1930, 18, 183.

22. Pizzarello, S.; Cronin, J.R. Non-racemic amino acids in the Murray and Murchison meteorites. Geochim. Cosmochim. Acta 2000, 64, 329-338.

23. Cronin, J.R.; Pizzarello, S. Enantiomeric excesses in meteoritic amino acids. Science 1997, 275, 951-955.

24. Pizzarello, S.; Huang, Y.S. Carbon isotopic analyses of individual Murchison amino acids. Geochim. Cosmochim. Acta 2003, 67, A380-A380.

25. Glavin, D.P.; Dworkin, J.P. Enrichment of the amino acid L-isovaline by aqueous alteration on CI and CM meteorite parent bodies. Proc. Natl. Acad. Sci. 2009, 106, 5487-5492.

26. Rubenstein, E.; Bonner, W.A.; Noyes, H.P.; Brown, G.S. Supernovae and life. Nature 1983, 306, 118.

27. Bonner, W.A.; Rubenstein, E. Supernovae, neutron stars and biomolecular chirality. BioSystems 1987, 20, 99-111.

28. Bailey, J.; Chrysostomou, A.; Hough, J.H.; Gledhill, T.M.; McCall, A.; Clark, S.; Menard, F.; Tamura, M. Circular polarization in star-formation regions: Implications for biomolecular homochirality. Science 1998, 281, 672-674. 
29. Bailey, J. Astronomical sources of circularly polarized light and the origin of homochirality. Orig. Life Evol. Biosph. 2001, 31, 167-183.

30. Buschermoehle, M.; Whittet, D.C.B.; Chrysostomou, A.; Hough, J.H.; Lucas, P.W.; Adamson, A.J.; Whitney, B.A.; Wolff, M.J. An extended search for circularly polarized infrared radiation from the OMC-1 region of orion. Astrophys. J. 2005, 624, 821-826.

31. Allamandola, L.J.; Bernstein, M.P.; Sandford, S.A.; Walker, R.L. Evolution of interstellar ices. Space Sci. Rev. 1999, 90, 219-232.

32. Bernstein, M.P.; Allamandola, L.J.; Sandford, S.A. Complex organics in laboratory simulations of interstellar/cometary ices. Adv. Space Res. 1997, 19, 991-998.

33. Nuevo, M.; Bredehoft, J.H.; Meierhenrich, U.; d'Hendecourt, L.; Thiemann, W. Urea, glycerol and glycolic acid in an organic residue produced by UV irradiation of interstellar/pre-cometary ice analogues. Astrobiology 2010, 10, 245-256.

34. Munoz Caro, G.M.; Meierhenrich, U.J.; Schutte, W.A.; Barbler, B.; Segovia, A.A.; Rosenbauer, H.; Thiemann, W.H.P.; Brack, A.; Greenberg, J.M. Amino acids from ultraviolet irradiation of interstellar ice analogues. Nature 2002, 416, 403-406.

35. Bernstein, M.P.; Dworkin, J.P.; Sandford, S.A.; Cooper, G.W.; Allamandola, L.J. Racemic amino acids from the ultraviolet photolysis of interstellar ice analogues. Nature 2002, 416, 401-403.

36. Nuevo, M.; Auger, G.; Blanot, D.; d'Hendecourt, L. A detailed study of the amino acids produced from the vacuum UV irradiation of interstellar ice analogs. Orig. Life Evol. Biosph. 2008, 38, 37-56.

37. Greenberg, J.M. What are comets made of? A model based on interstellar dust. In Comets; University of Arizona Press: Tucson, AZ, USA, 1982; pp. 131-163.

38. Greenberg, J.M. Chemical evolution in space. Orig. Life 1984, 24, 25-36.

39. Greenberg, J.M. Chirality in interstellar dust and in comets: Life from dead stars. AIP Conf. Proc. 1996, 379, 185-210.

40. Chyba, C.; Sagan, C. Endogenous production, exogenous delivery and impact-shock synthesis of organic molecules: An inventory for the origins of life. Nature 1992, 355, 125-132.

41. Oro, J. Comets and formation of biochemical compounds on primitive earth. Nature 1961, 190, 389-390.

42. Greenberg, J.M. The structure and evolution of interstellar grains. Sci. Am. 1984, 250, 124-154.

43. Takenaka, N. Recent Developments of Chemistry and Photochemistry in Ices; Transworld Research Network: Kerala, India, 2008; p. 202.

44. Agarwal, V.K.; Schutte, W.; Greenberg, J.M.; Ferris, J.P.; Briggs, R.; Connor, S.; Van de Bult, C.P.E.M.; Baas, F. Photochemical reactions in interstellar grains photolysis of carbon monoxide, ammonia, and water. Orig. Life 1985, 16, 21-40.

45. Ehrenfreund, P.; Charnley, S.B. Organic molecules in the interstellar medium, comets, and meteorites: A voyage from dark clouds to the early earth. Annu. Rev. Astron. Astrophys. 2000, 38, 427-483.

46. Irvine, W.M. The molecular composition of dense interstellar clouds. NATO ASI Ser. Ser. C 1991, 323, 89-121.

47. Chiar, J.E. The nature and evolution of interstellar ices. Orig. Life Evol. Biosph. 1997, 27, 79-100.

48. Greenberg, J.M. Radical formation, chemical processing, and explosion of interstellar grains. Astrophys. Space Sci. 1976, 39, 9-18. 
49. Duley, W.W. Chemical evolution of carbonaceous material in interstellar clouds. Astrophys. J. 2000, 528, 841-848.

50. Ehrenfreund, P.; Kerkhof, O.; Schutte, W.A.; Boogert, A.C.A.; Gerakines, P.A.; Dartois, E.; D'Hendecourt, L.; Tielens, A.G.G.M.; Van Dishoeck, E.F.; Whittet, D.C.B. Laboratory studies of thermally processed $\mathrm{H}_{2} \mathrm{O}-\mathrm{CH}_{3} \mathrm{OH}-\mathrm{CO}_{2}$ ice mixtures and their astrophysical implications. Astron. Astrophys. 1999, 350, 240-253.

51. Gibb, E.L.; Whittet, D.C.B.; Boogert, A.C.A.; Tielens, A.G.G.M. Interstellar ice: The infrared space observatory legacy. Astrophys. J. Suppl. Ser. 2004, 151, 35-73.

52. Cosmic rays are charged high energetic particles which are known to create secondary electrons capable of triggering chemical reactions.

53. DHendecourt, L.B.; Allamandola, L.J.; Baas, F.; Greenberg, J.M. Inter-stellar grain explosions-molecule cycling between gas and dust. Astron. Astrophys. 1982, 109, L12-L14.

54. Cronin, J.R.; Pizzarello, S. Amino acids in meteorites. Adv. Space Res. 1983, 3, 5-18.

55. Kvenvolden, K.A.; Lawless, J.; Pering, K.; Peterson, E.; Flores, J.; Ponnamperuma, C.; Kaplan, I.R.; Moore, C. Evidence for extraterrestrial amino-acids and hydrocarbons in the Murchison meteorite. Nature 1970, 228, 923-926.

56. Takano, Y.; Kaneko, T.; Kobayashi, K.; Hiroishi, D.; Ikeda, H.; Marumo, K. Experimental verification of photostability for free- and bound-amino acids exposed to gamma-rays and UV irradiation. Earth Planets Space 2004, 56, 669-674.

57. Elsila, J.E.; Dworkin, J.P.; Bernstein, M.P.; Martin, M.P.; Sandford, S.A. Mechanisms of amino acid formation in interstellar ice analogs. Astrophys. J. 2007, 660, 911-918.

58. Briggs, R.; Ertem, G.; Ferris, J.P.; Greenberg, J.M.; McCain, P.J.; Mendoza-Gomez, C.X.; Schutte, W. Comet Halley as an aggregate of interstellar dust and further evidence for the photochemical formation of organics in the interstellar medium. Orig. Life Evol. Biosph. 1992, 22, 287-307.

59. Takahashi, J.-I.; Masuda, H.; Kaneko, T.; Kobayashi, K.; Saito, T.; Hosokawa, T. Photochemical abiotic synthesis of amino-acid precursors from simulated planetary atmospheres by vacuum ultraviolet light. J. Appl. Phys. 2005, 98, 024907.1-024907.6.

60. Cottin, H.; Szopa, C.; Moore, M.H. Production of hexamethylenetetramine in photolyzed and irradiated interstellar cometary ice analogs. Astrophys. J. 2001, 561, L139-L142.

61. Kobayashi, K.; Kaneko, T.; Saito, T. Characterization of complex organic compounds formed in simulated planetary atmospheres by the action of high energy particles. Adv. Space Res. 1999, 24, 461-464.

62. Takahashi, J.-I.; Hosokawa, T.; Masuda, H.; Kaneko, T.; Kobayashi, K.; Saito, T.; Utsumi, Y. Abiotic synthesis of amino acids by x-ray irradiation of simple inorganic gases. Appl. Phys. Lett. 1999, 74, 877-879.

63. Takano, Y.; Masuda, H.; Kaneko, T.; Kobayashi, K. Formation of amino acids from possible interstellar media by gamma-rays and UV irradiation. Chem. Lett. 2002, 986-987.

64. Takano, Y.; Marumo, K.; Yabashi, S.; Kaneko, T.; Kobayashi, K. Pyrolysis of complex organics following high-energy proton irradiation of a simple inorganic gas mixture. Appl. Phys. Lett. 2004, $85,1633-1635$. 
65. Cronin, J.R.; Chang, S. Organic matter in meteorites: Molecular and isotopic analyses of the Murchison meteorite. In Chemistry of Life's Origins (NATO ASI Ser.); Greenberg, J.M., Mendoza-Gomez, C.X., Pirronello, V., Eds; Kluwer: Dordrecht, The Netherlands, 1993; Volume 416, pp. 209-258.

66. Most of the components can be obtained by extracting meteorite powders with polar and nonpolar solvents. Nevertheless, the quantities of detectable amino acids increase dramatically when using acid hydrolysis. Hydrolytic breakdown of small polypeptides or acid-labile amino acid precursors are assumed to account for these increases [65].

67. Stoks, P.G.; Schwartz, A.W. Uracil in carbonaceous meteorites. Nature 1979, 282, 709-710.

68. Stoks, P.G.; Schwartz, A.W. Basic nitrogen-heterocyclic compounds in the Murchison meteorite. Geochim. Cosmochim. Acta 1982, 46, 309-315.

69. Meierhenrich, U.J.; Caro, G.M.M.; Bredehoft, J.H.; Jessberger, E.K.; Thiemann, W.H.P. Identification of diamino acids in the Murchison meteorite. Proc. Natl. Acad. Sci. 2004, 101, 9182-9186.

70. Martins, Z.; Botta, O.; Fogel, M.L.; Sephton, M.A.; Glavin, D.P.; Watson, J.S.; Dworkin, J.P.; Schwartz, A.W.; Ehrenfreund, P. Extraterrestrial nucleobases in the Murchison meteorite. Orig. Life Evol. Biosph. 2009, 39, 214-214.

71. Pizzarello, S.; Huang, Y.S.; Fuller, M. The carbon isotopic distribution of Murchison amino acids. Geochim. Cosmochim. Acta 2004, 68, 4963-4969.

72. Bredehoft, J.H.; Meierhenrich, U. Amino Acid Structures from UV Irradiation of Simulated Interstellar Ices; Transworld Research Network: Kerala, India, 2008; pp. 175-202.

73. Balavoine, G.; Moradpour, A.; Kagan, H.B. Preparation of chiral compounds with high optical purity by irradiation with circularly polarized light, A model reaction for the prebiotic generation of optical activity. J. Am. Chem. Soc. 1974, 96, 5152-5158.

74. Nishino, H.; Kosaka, A.; Hembury, G.A.; Matsushima, K.; Inoue, Y. The pH dependence of the anisotropy factors of essential amino acids. J. Chem. Soc. Perkin Trans. 2 2002, 582-590.

75. Brückner, H.; Maisch, J.; Reinecke, C.; Kimonyo, A. Use of $\alpha$-aminoisobutyric acid and isovaline as marker amino acids for the detection of fungal polypeptide antibiotics. Screen. Hypocrea. Amino Acids 1991, 1, 251-257.

76. Bruckner, H.; Becker, D.; Gams, W.; Degenkolb, T. Aib and Iva in the biosphere: Neither rare nor necessarily extraterrestrial. Chem. Biodivers. 2009, 6, 38-56.

77. Engel, M.H.; Macko, S.A.; Silfer, J.A. Carbon isotope composition of individual amino-acids in the Murchison meteorite. Natsure 1990, 348, 47-49.

78. Epstein, S.; Krishnamurthy, R.V.; Cronin, J.R.; Pizzarello, S.; Yuen, G.U. Unusual stable isotope ratios in amino-acid and carboxylic-acid extracts from the Murchison meteorite. Nature 1987, $326,477-479$.

79. Yuen, G.; Blair, N.; Desmarais, D.J.; Chang, S. Carbon isotope composition of low-molecular weight hydrocarbons and monocarboxylic acids from Murchison meteorite. Nature 1984, 307, 252-254.

80. Engel, M.H.; Macko, S.A. Isotopic evidence for extraterrestrial non-racemic amino acids in the Murchison meteorite. Nature 1997, 389, 265-268. 
81. Pizzarello, S.; Feng, X.; Epstein, S.; Cronin, J.R. Isotopic analyses of nitrogenous compounds from the Murchison meteorite-Ammonia, Amines, Amino-Acids, and Polar Hydrocarbons. Geochim. Cosmochim. Acta 1994, 58, 5579-5587.

82. Pizzarello, S.; Huang, Y.S. The deuterium enrichment of individual amino acids in carbonaceous meteorites: A case for the presolar distribution of biomolecule precursors. Geochim. Cosmochim. Acta 2005, 69, 599-605.

83. Pizzarello, S.; Krishnamurthy, R.V.; Epstein, S.; Cronin, J.R. Isotopic analyses of amino-acids from the Murchison meteorite. Geochim. Cosmochim. Acta 1991, 55, 905-910.

84. Cohen, B.A.; Chyba, C.F. Racemization of meteoritic amino acids. Icarus 2000, 145, $272-281$.

85. Cronin, J.R.; Moore, C.B. Amino acid analyses of Murchison, Murray, and Allende carbonaceous chondrites. Science 1971, 172, 1327-1328.

86. Ehrenfreund, P.; Glavin, D.P.; Botta, O.; Cooper, G.; Bada, J.L. Extraterrestrial amino acids in Orgueil and Ivuna: Tracing the parent body of $\mathrm{Cl}$ type carbonaceous chondrites. Proc. Natl. Acad. Sci. USA 2001, 98, 2138-2141.

87. Nielsen, P.E. Peptide Nucleic-Acid (PNA)-A model structure for the primordial genetic material. Orig. Life Evol. Biosph. 1993, 23, 323-327.

88. Nielsen, P.E.; Egholm, M.; Berg, R.H.; Buchardt, O. Sequence-selective recognition of DNA by strand displacement with a thymine-substituted polyamide. Science 1991, 254, 1497-1500.

89. Pizzarello, S.; Weber, A.L. Stereoselective syntheses of pentose sugars under realistic prebiotic conditions. Orig. Life Evol. Biosph. 2010, 40, 3-10.

90. Weber, A.L.; Pizzarello, S. The peptide-catalyzed stereospecific synthesis of tetroses: A possible model for prebiotic molecular evolution. Proc. Natl. Acad. Sci. USA. 2006, 103, 12713-12717.

91. Bada, J.L.; Miller, S.L. Racemization and the origin of optically-active organic-compounds in living organisms. Biosystems 1987, 20, 21-26.

92. Abe, I.; Fujimoto, N.; Nishiyama, T.; Terada, K.; Nakahara, T. Rapid analysis of amino acid enantiomers by chiral-phase capillary gas chromatography. J. Chromatogr. A 1996, 722, 221-227.

93. Peltzer, E.T.; Bada, J.L.; Schlesinger, G.; Miller, S.L. The chemical conditions on the parent body of the Murchison meteorite: some conclusions based on amino, hydroxy and dicarboxylic acids. Adv. Space Res. 1984, 4, 69-74.

94. Cronin, J.R.; Cooper, G.W.; Pizzarello, S. Characteristics and formation of amino acids and hydroxy acids of the Murchison meteorite. Adv. Space Res. 1995, 15, 91-97.

95. Botta, O.; Glavin, D.P.; Kminek, G.; Bada, J.L. Relative amino acid concentrations as a signature for parent body processes of carbonaceous chondrites. Orig. Life Evol. Biosph. 2002, 32, 143-163.

96. Crovisier, J.; Bockelee-Morvan, D. Remote observations of the composition of cometary volatiles. Space Sci. Rev. 1999, 90, 19-32.

97. Podolak, M.; Mekler, Y. Dirty ice grains in the protoplanetary nebula. Planet Space Sci. 1997, 45, 1401-1406.

98. Blagojevic, V.; Petrie, S.; Bohme, D.K. Gas-phase syntheses for interstellar carboxylic and amino acids. Mon. Not. R. Astron. Soc. 2003, 339, L:7-L:11.

99. Largo, A.; Redondo, P.; Barrientos, C. Theoretical study of possible ion-molecule reactions leading to precursors of glycine in the interstellar medium. Int. J. Quantum Chem. 2004, 98, 355-360. 
100. Chakrabarti, S.; Chakrabarti, S.K. Can DNA bases be produced during molecular cloud collapse? Astron. Astrophys. 2000, 354, L6-L8.

101. Millar, T.J.; Bennett, A.; Herbst, E. Deuterium fractionation in dense interstellar clouds. Astrophys. J. 1989, 340, 906-920.

102. Inoue, Y. Asymmetric photochemical reactions in solution. Chem. Rev. 1992, 92, 741-770.

103. Flores, J.J.; Bonner, W.A.; Massey, G.A. Asymmetric photolysis of (RS)-leucine with circularly polarized ultraviolet light. J. Am. Chem. Soc. 1977, 99, 3622-3625.

104. Nishino, H.; Kosaka, A.; Hembury, G.A.; Shitomi, H.; Onuki, H.; Inoue, Y. Mechanism of $\mathrm{pH}$-dependent photolysis of aliphatic amino acids and enantiomeric enrichment of racemic leucine by circularly polarized light. Org. Lett. 2001, 3, 921-924.

105. Naito, Y.; Nishino, H.; Inoue, Y. The absolute asymmetric synthesis by two-photon excitation using femtosecond circularly polarized pulse. In proceedings of the Photochirogenesis Symposium, Osaka, Japan, September, 2001.

106. Bonner, W.A.; Bean, B.D. Asymmetric photolysis with elliptically polarized light. Orig. Life Evol. Biosph. 2000, 30, 513-517.

107. Donovan, J.W.; Mapes, C.J.; Davis, J.G.; Hamburg, R.D. Dissociation of chicken egg-white macroglobulin into subunits in acid. Hydrodynamic, spectrophotometric, and optical rotatory measurements. Biochemistry 1969, 8, 4190-4199.

108. Meierhenrich, U.J.; Nahon, L.; Alcaraz, C.; Bredehoft, J.H.; Hoffmann, S.V.; Barbier, B.; Brack, A. Asymmetric vacuum UV photolysis of the amino acid leucine in the solid state. Angew. Chem. Int. Ed. 2005, 44, 5630-5634.

109. Meierhenrich, U.; Filippi, J.J.; Meinert, C.; Hoffmann, S.V.; Bredehoft, J.H.; Nahon, L. Photolysis of rac-leucine with circularly polarized synchrotron radiation. Chem. Biodivers. 2010, 7, in press.

110. Kagan, H.; Moradpour, A.; Nicoud, J.F.; Balavoine, G.; Martin, R.H.; Cosyn, J.P. Photochemistry with circularly polarized light. II) Asymmetric synthesis of octa and nonahelicene. Tetrahedron Lett. 1971, 12, 2479-2482.

111. Rau, H. Asymmetric photochemistry in solution. Chem. Rev. 1983, 83, 535-547.

112. Takano, Y.; Takahashi, J.; Kaneko, T.; Marumo, K.; Kobayashi, K. Asymmetric synthesis of amino acid precursors in interstellar complex organics by circularly polarized light. Earth Planet. Sci. Lett. 2007, 254, 106-114.

113. Nuevo, M.; Meierhenrich, U.J.; d'Hendecourt, L.; Caro, G.M.M.; Dartois, E.; Deboffle, D.; Thiemann, W.H.P.; Bredehoft, J.H.; Nahon, L. Enantiomeric separation of complex organic molecules produced from irradiation of interstellar/circumstellar ice analogs. Adv. Space Res. 2007, 39, 400-404.

114. Nuevo, M.; Meierhenrich, U.J.; Caro, G.M.M.; Dartois, E.; d'Hendecourt, L.; Deboffle, D.; Auger, G.; Blanot, D.; Bredehoft, J.H.; Nahon, L. The effects of circularly polarized light on amino acid enantiomers produced by the UV irradiation of interstellar ice analogs. Astron. Astrophys. 2006, 457, 741-751.

115. Cronin, J.R.; Pizzarello, S. Amino Acid Enantiomer Excesses in Meteorites: Origin and Significance. In Life Sciences: Exobiology; Raulin, F., Kobayashi, K., Brack, A., Eds.; Pergamon Press Ltd.: Oxford, UK, 1999; Volume 23, pp. 293-299. 
116. Takahashi, J.; Shinojima, H.; Seyama, M.; Ueno, Y.; Kaneko, T.; Kobayashi, K.; Mita, H.; Adachi, M.; Hosaka, M.; Katoh, M. Chirality emergence in thin solid films of amino acids by polarized light from synchrotron radiation and free electron laser. Int. J. Mol. Sci. 2009, 10, 3044-3064.

117. Pasek, M.; Lauretta, D. Extraterrestrial flux of potentially prebiotic C, N, and P to the early earth. Orig. Life Evol. Biosph. 2008, 38, 5-21.

118. Huebner, W.F.; Boice, D.C. Comets as a possible source of prebiotic molecules. Orig. Life Evol. Biosph. 1992, 21, 299-315.

119. Brownlee, D.; Tsou, P.; Aleon, J.; Alexander, C.M.O'D.; Araki, T.; Bajt, S.; Baratta, G.A.; Bastien, R.; Bland, P.; Bleuet, P. et al. Comet 81P/Wild 2 under a microscope. Science 2006, 314, 1711-1716.

120. Goesmann, F.; Rosenbauer, H.; Roll, R.; Szopa, C.; Raulin, F.; Sternberg, R.; Israel, G.; Meierhenrich, U.; Thiemann, W.; Munoz-Caro, G. COSAC, The cometary sampling and composition experiment on philae. Space Sci. Rev. 2007, 128, 257-280.

121. Thiemann, W.H.P.; Meierhenrich, U. ESA mission ROSETTA will probe for chirality of cometary amino acids. Orig. Life Evol. Biosph. 2001, 31, 199-210.

122. Greenberg, J.M.; Yencha, A.J.; Corbett, J.W.; Frisch, H.L. Ultraviolet effects on the chemical composition and optical properties of interstellar grains. Mem. Soc. Roy. Sci. 1972, 3, 425-436.

123. Caro, G.M.M.; Meierhenrich, U.; Schutte, W.A.; Thiemann, W.H.P.; Greenberg, J.M. UV-photoprocessing of interstellar ice analogs: Detection of hexamethylenetetramine-based species. Astron. Astrophys. 2004, 413, 209-216.

124. Kuan, Y.-J.; Charnley, S.B.; Huang, H.-C.; Tseng, W.-L.; Kisiel, Z. Interstellar glycine. Astrophys. J. 2003, 593, 848-867.

125. Snyder, L.E.; Lovas, F.J.; Hollis, J.M.; Friedel, D.N.; Jewell, P.R.; Remijan, A.; Ilyushin, V.V.; Alekseev, E.A.; Dyubko, S.F. A rigorous attempt to verify interstellar glycine. Astrophys. J. 2005, 619, 914-930.

126. Ehrenfreund, P.; Bernstein, M.P.; Dworkin, J.P.; Sandford, S.A.; Allamandola, L.J. The photostability of amino acids in space. Astrophys. J. 2001, 550, L95-L99.

127. Elsila, J.E.; Glavin, D.P.; Dworkin, J.P. Cometary glycine detected in samples returned by Stardust. Meteorit. Planet. Sci. 2009, 44, 1323-1330.

128. Fukue, T.M., T.; Kandori, R.; Kusukabe, N.; Hough, J.H.; Bailey, J.; Whittet, D.C.B.; Lucas, P.W.; Nakajima, Y.; Hashimoto, J. Extended high circular polarization in the orion massive star forming region: Implications for the origin of homochirality in the solar system. Orig. Life Evol. Biosph. 2010, 40, 335-346.

129. Menard, F.; Chrysostomou, A.; Gledhill, T.; Hough, J.H.; Bailey, J. High circular polarization in the star forming region NGC 6334: Implications for biomoleclar homochirality. Astron. Soc. Pac. Conf. Ser. 2000, 213, 355-358.

130. Lucas, P.W.; Hough, J.H.; Bailey, J.; Chrysostomou, A.; Gledhill, T.M.; McCall, A. UV circular polarisation in star formation regions: The origin of homochirality? Orig. Life Evol. Biosph. 2005, 35, 29-60.

131. Mason, S.F. Extraterrestrial handedness. Nature 1997, 389, 804. 
132. Jordan, I.K.; Kondrashov, F.A.; Adzhubei, I.A.; Wolf, Y.I.; Koonin, E.V.; Kondrashov, A.S.; Sunyaev, S. A universal trend of amino acid gain and loss in protein evolution. Nature. 2005, 433, 633-638.

133. Cerf, C.; Jorissen, A. Is amino-acid homochirality due to asymmetric photolysis in space? Space Sci. Rev. 2000, 92, 603-612.

134. Sephton, M.A.; Verchovsky, A.B.; Bland, P.A.; Gilmour, I.; Grady, M.M.; Wright, I.P. Investigating the variations in carbon and nitrogen isotopes in carbonaceous chondrites. Geochim. Cosmochim. Acta 2003, 67, 2093-2108.

135. Engrand, C.; Maurette, M. Carbonaceous micrometeorites from Antarctica. Meteorit. Planet. Sci. 1998, 33, 565-580.

136. Matrajt, G.; Pizzarello, S.; Taylor, S.; Brownlee, D. Concentration and variability of the AIB amino acid in polar micrometeorites: Implications for the exogenous delivery of amino acids to the primitive earth. Meteorit. Planet. Sci. 2004, 39, 1849-1858.

137. Botta, O.; Glavin, D.P.; Dworkin, J.P.; Matrajt, G.; Harvey, R.P. Detection of AIB in antarctic ice samples: Implications for exogenous delivery of prebiotic organic compounds. Orig. Life Evol. Biosph. 2009, 39, 225-226.

138. Fenwick, D.R.; Kagan, H.B. Asymmetric amplification. Top. Stereochem. 1999, 22, 257-296.

139. Shibata, T.; Yamamoto, J.; Matsumoto, N.; Yonekubo, S.; Osanai, S.; Soai, K. Amplification of a slight enantiomeric imbalance in molecules based on asymmetric autocatalysis: The first correlation between high enantiomeric enrichment in a chiral molecule and circularly polarized light. J. Am. Chem. Soc. 1998, 120, 12157-12158.

140. Soai, K.; Shibata, T.; Morioka, H.; Choji, K. Asymmetric autocatalysis and amplification of enantiomeric excess of a chiral molecule. Nature 1995, 378, 767-768.

141. Soai, K.; Shibata, T.; Sato, I. Enantioselective automultiplication of chiral molecules by asymmetric autocatalysis. Acc. Chem. Res. 2000, 33, 382-390.

142. Reiner, C.; Nicholson, G.J.; Nagel, U.; Schurig, V. Evaluation of enantioselective gas chromatography for the determination of minute deviations from racemic composition of amino acids with emphasis on tyrosine: accuracy and precision of the method. Chirality 2007, 19, 401-414.

(C) 2010 by the authors; licensee MDPI, Basel, Switzerland. This article is an Open Access article distributed under the terms and conditions of the Creative Commons Attribution license (http://creativecommons.org/licenses/by/3.0/). 